\title{
Flattening functions on flowers
}

\author{
EDMUND HARRISS and OLIVER JENKINSON \\ School of Mathematical Sciences, Queen Mary, University of London, Mile End Road, \\ London E1 4NS, UK \\ (e-mail: edmund.harriss@mathematicians.org.uk,omj@maths.qmul.ac.uk)
}

(Received 1 June 2006 and accepted in revised form 27 February 2007)

\begin{abstract}
Let $T$ be an orientation-preserving Lipschitz expanding map of the circle $\mathbb{T}$. A pre-image selector is a map $\tau: \mathbb{T} \rightarrow \mathbb{T}$ with finitely many discontinuities, each of which is a jump discontinuity, and such that $\tau(x) \in T^{-1}(x)$ for all $x \in \mathbb{T}$. The closure of the image of a pre-image selector is called a flower and a flower with $p$ connected components is called a $p$-flower. We say that a real-valued Lipschitz function can be Lipschitz flattened on a flower whenever it is Lipschitz cohomologous to a constant on that flower. The space of Lipschitz functions which can be flattened on a given $p$-flower is shown to be of codimension $p$ in the space of all Lipschitz functions, and the linear constraints determining this subspace are derived explicitly. If a Lipschitz function $f$ has a maximizing measure $S$ which is Sturmian (i.e. is carried by a 1-flower), it is shown that $f$ can be Lipschitz flattened on some 1-flower carrying $S$.
\end{abstract}

\section{Introduction}

This paper is concerned with certain special representatives of dynamically defined cohomology classes, mainly motivated by the theory of maximizing measures (see e.g. [Bou1, Bou2, CLT, Jen2] for some background to this area). Given a continuous selfmap $T: X \rightarrow X$ of a compact metric space $X$, let $\mathcal{M}_{T}$ denote the set of $T$-invariant Borel probability measures. For a continuous function $f: X \rightarrow \mathbb{R}$, a measure $\mu \in \mathcal{M}_{T}$ is called maximizing if $\int f d \mu=\max _{m \in \mathcal{M}_{T}} \int f d m$.

One method of determining the maximizing measure(s) for a function $f$ consists of finding a continuous function $\varphi$ such that the set $M(\tilde{f}):=\tilde{f}^{-1}(\max \tilde{f})$ of maxima of $\tilde{f}:=$ $f+\varphi-\varphi \circ T$ carries $\dagger$ at least one $T$-invariant measure. The maximizing measures for $f$ (and for $\tilde{f}$ ) are then precisely those invariant measures carried by $M(\tilde{f})$. It is known that such $\varphi$ always exist if the map $T$ has some hyperbolicity and there is an appropriate control on the modulus of continuity of the function $f$ (see e.g. [Bou1, Bou2, CG, CLT, Jen2]).

$\dagger$ We say that a subset $G \subset X$ carries a measure $\mu$ if the (topological) support of $\mu$, which we always denote by $\operatorname{supp}(\mu)$, is contained in $G$. 
If $T$ is an expanding map, and $f$ is Lipschitz, the function $\varphi$ may be chosen so that $T(M(\tilde{f}))=X$, i.e. the set of maxima of $\tilde{f}$ contains at least one pre-image of every point in $X$ (see [Bou2, Theorem 1]). In this case, it is easily seen that $M(\tilde{f})$ carries at least one invariant measure. In certain situations, it may be possible to choose $\tilde{f}$ such that $M(\tilde{f})$ contains precisely one pre-image of every point in $X$; in this case we may think of the function $f$ as determining a particular selection of pre-images of $T$. The topology of the space $X$ may, however, preclude $M(\tilde{f})$ from containing exactly one pre-image of every point; for example this is the case if $X=\mathbb{T}=\mathbb{R} / \mathbb{Z}$ is the circle, where no closed subset $F \subset \mathbb{T}$ is mapped bijectively onto $\mathbb{T}$ by an expanding map $T$. Nevertheless, it may be the case that, with finitely many exceptions, every point in $\mathbb{T}$ has a unique pre-image in $F=M(\tilde{f})$. For example if $T: \mathbb{T} \rightarrow \mathbb{T}$ is defined by $T(x)=2 x(\bmod 1)$ and $F$ is any closed semi-circle, then, apart from the common image of the endpoints of $F$, every point in $\mathbb{T}$ has a unique pre-image in $F$. More generally we may choose $F$ to be the union of a finite number of disjoint closed intervals with the property that the only points in $\mathbb{T}$ without a unique pre-image in $F$ are the images of the boundary points in $F$. Such sets $F$ have been studied by Brémont [Br1, Br2], who called them (finite) flowers (some examples of flowers are depicted in Figure 1 in $§ 2)$.

When $T(x)=2 x(\bmod 1)$, Bousch [Bou1] has shown that if $f: \mathbb{T} \rightarrow \mathbb{R}$ is any trigonometric polynomial of degree one then indeed there exists $\tilde{f}=f+\varphi-\varphi \circ T$ such that $M(\tilde{f})$ is a closed semi-circle (i.e. $f$ satisfies the so-called Sturmian condition, cf. [Bou1]). Since closed semi-circles have the notable property of carrying one, and only one, $T$-invariant measure (so-called Sturmian measures, see e.g. [BM, BS]), he deduced that every degree-one trigonometric polynomial has a unique maximizing measure, and that this measure is Sturmian. The analogous result has been obtained in [ADJR] for a certain family of piecewise linear functions, and experimental evidence suggests that Sturmian maximizing measures appear rather often for sufficiently simple functions $f$.

If a Lipschitz function $f$ is known to have a Sturmian maximizing measure $\dagger$ then a natural problem is to determine precisely which of the Sturmian measures is $f$-maximizing. We will establish (see Theorem 4.9) the following useful necessary condition for a Sturmian measure $S$ to be $f$-maximizing: there exists a closed semi-circle $F$ carrying $S$, and a Lipschitz function $\varphi$, such that the restriction of $\tilde{f}=f+\varphi-\varphi \circ T$ to $F$ is a constant function. This condition was introduced by Bousch [Bou1], who referred to it as the pre-Sturmian condition since it is clearly implied by his Sturmian condition mentioned above. The content of Theorem 4.9 is that, for Lipschitz $f$ whose maximizing measure is Sturmian yet which do not satisfy the Sturmian condition (such $f$ exist, cf. Example 4.12), the pre-Sturmian condition is still satisfied (on some semi-circle carrying the maximizing measure).

The practical utility of Theorem 4.9 stems from the fact that, as noted by Bousch, solving the pre-Sturmian condition for $F$ amounts to solving a real-valued equation. This real-valued equation, which appears explicitly in [Bou1], and as a particular case of our Theorem 3.6, can be solved numerically (cf. Remark 4.10). It should be contrasted to the functional equation (namely, $\varphi(x)+\left(\max _{m \in \mathcal{M}_{T}} \int f d m\right)=\max _{y \in T^{-1}(x)}(f+\varphi)(y)$, 
cf. [Bou1]) which, a priori, must be solved for $\varphi$ in order to check the Sturmian condition (which in any case may not be satisfied, cf. Example 4.12).

In the above discussion of the Sturmian and pre-Sturmian conditions the expanding map was assumed, for simplicity, to be $T(x)=2 x(\bmod 1)$. In fact, Theorem 4.9 is formulated in terms of arbitrary (orientation-preserving) Lipschitz expanding maps of the circle. In this general setting, closed semi-circles are replaced by 1 -flowers (i.e. flowers with a single connected component). Our interest in the rest of the paper is in more general $p$-flowers (i.e. flowers with $p$ connected components) for orientation-preserving Lipschitz expanding circle maps. These are formally introduced in $\S 2$, in terms of pre-image selectors (roughly, a pre-image selector is an inverse map for $\left.T\right|_{F}$ ). In $\S 3$, we consider the generalization of the pre-Sturmian condition to arbitrary $p$-flowers $F$ : for a Lipschitz function $f$, if there exists a Lipschitz $\varphi$ such that $\left.(f+\varphi-\varphi \circ T)\right|_{F}$ is a constant function, we say that $f$ is Lipschitz flattened on $F$ (see Figure 2 in $\S 3$ ). Theorem 3.6 characterizes this situation in terms of an explicit vector-valued equation in $\mathbb{R}^{p}$. As with the real-valued equation arising from the pre-Sturmian condition, this vector-valued equation can in practice be solved numerically in order to determine those $p$-flowers $F$ on which $f$ can be Lipschitz flattened.

Although nonlinear in $F$, the $p$ constraints detailed in Theorem 3.6 depend linearly on the Lipschitz function $f$. Theorem 3.8 asserts that these constraints are independent; in other words, the space $\operatorname{Lip}_{F}$ of Lipschitz functions which can be Lipschitz flattened on a given $p$-flower $F$ is of codimension $p$ in the space of all Lipschitz functions.

\section{Pre-image selectors and flowers}

We consider the circle $\mathbb{T}$ as the space $\mathbb{R} / \mathbb{Z}$, i.e. as the quotient of the additive group of real numbers by the subgroup of integers. The usual distance function on $\mathbb{R}$ induces a quotient distance function on $\mathbb{T}$, which we shall denote by $d$. The usual orientation on $\mathbb{R}$ induces an orientation on $\mathbb{T}$. If $a, b \in \mathbb{T}$ then $[a, b]$ denotes the positively oriented closed arc connecting $a$ to $b$, and is called a closed interval. Open intervals $(a, b)$, and half-open intervals $[a, b)$ and $(a, b]$ are defined analogously, in accordance with the usual notational conventions. We shall refer to $a$ (respectively $b$ ) as the left (respectively right) endpoint of any such interval. For $a=c+\mathbb{Z} \in \mathbb{T}$, let $i: \mathbb{T} \rightarrow \mathbb{R}$ be the unique map with image $[c, c+1)$ such that $i(u) \in u$ for all $u \in \mathbb{T}$. Let $<_{a}$ denote the ordering on $\mathbb{T}$ induced by the usual ordering on $[c, c+1)$, i.e. $u<_{a} v$ if and only if $i(u)<i(v)$.

A continuous map $T: \mathbb{T} \rightarrow \mathbb{T}$ is expanding if there exists $\varepsilon>0$ and $K>1$ such that, for all $x, y \in \mathbb{T}$,

$$
d(x, y)<\varepsilon \quad \Rightarrow \quad d(T(x), T(y)) \geq K d(x, y) .
$$

The degree of an expanding map is an integer of absolute value at least 2 (see e.g. [KH, p. 73]). In this paper, it will be notationally convenient to consider only expanding maps which are orientation-preserving, i.e. which have degree $k \geq 2$. This, however, is not an essential restriction: results analogous to those in this paper hold for orientation-reversing expanding maps, and can be proved via slight modifications of the proofs given here. Any expanding map of degree $k$ is topologically conjugate to the map $T_{k}(x)=k x(\bmod 1)($ see e.g. [KH, p. 73, Theorem 2.4.6]); it will be useful to keep in mind the maps $T_{k}$ as concrete examples for the notions and results of this paper. 


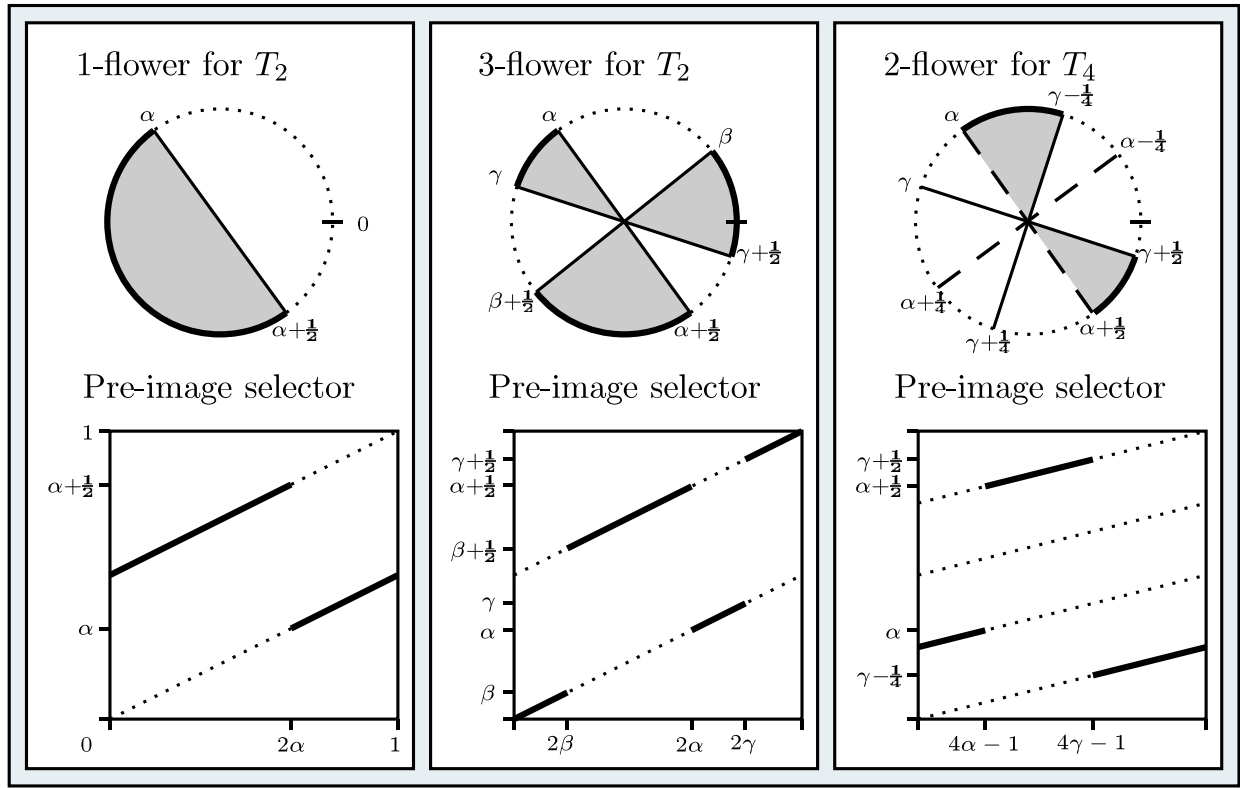

FIGURE 1. Flowers and their pre-image selectors (see Definition 2.2).

If the expanding map $T: \mathbb{T} \rightarrow \mathbb{T}$ has degree $k \geq 2$, and $a_{0}=T\left(a_{0}\right)$ is one of its $k-1$ fixed points, then write $T^{-1}\left(a_{0}\right)=\left\{a_{0}, \ldots, a_{k-1}\right\}$, where $a_{0}<\cdots<a_{k-1}$. Define $X_{k-1}=\left[a_{k-1}, a_{0}\right)$, and $X_{i}=\left[a_{i}, a_{i+1}\right)$ for $0 \leq i \leq k-2$. For $0 \leq i \leq k-1$, the map

$$
T_{i}:=\left(\left.T\right|_{X_{i}}\right)^{-1}
$$

is called an inverse branch of $T$.

We shall mainly be concerned with expanding maps $T$ which are in addition Lipschitz continuous, i.e. there exists $C>0$ such that

$$
d(T x, T y) \leq C d(x, y) \text { for all } x, y \in \mathbb{T} .
$$

Let Leb denote normalized Lebesgue measure on $\mathbb{T}$, and let $L^{1}$ denote the space of real-valued functions on $\mathbb{T}$ which are integrable with respect to Leb. For $g \in L^{1}$ we write $\int g$ to denote the integral of $g$ with respect to Leb. Let $L^{\infty}$ denote the space of functions $g: \mathbb{T} \rightarrow \mathbb{R}$ which are essentially bounded with respect to Leb, and let $\|g\|_{L^{\infty}}$ denote the essential supremum of $|g|$. Let $L_{0}^{\infty}=\left\{g \in L^{\infty}: \int g=0\right\}$. The following well-known result (see e.g. [Zie, Theorem 2.2.1]) will be used frequently.

LEMMA 2.1. If $U: \mathbb{T} \rightarrow \mathbb{T}$ is piecewise Lipschitz, then its derivative $U^{\prime}$ exists Lebesgue almost everywhere, and defines an element of $L^{\infty}$.

If $U: \mathbb{T} \rightarrow \mathbb{T}$ is Lipschitz continuous then $U^{\prime}$ belongs to $L_{0}^{\infty}$. Conversely, every element of $L_{0}^{\infty}$ is the derivative of a Lipschitz continuous function on $\mathbb{T}$.

An orientation-preserving Lipschitz map $T: \mathbb{T} \rightarrow \mathbb{T}$ is easily seen to be expanding if and only if there exists $K>1$ such that $T^{\prime}(x) \geq K$ for Lebesgue almost every $x \in \mathbb{T}$. 
Definition 2.2. Let $T: \mathbb{T} \rightarrow \mathbb{T}$ be an expanding map. A pre-image selector for $T$ is a map $\tau: \mathbb{T} \rightarrow \mathbb{T}$ such that:

(i) $\quad \tau(x) \in T^{-1}(x)$ for all $x \in \mathbb{T}$;

(ii) $\tau$ has finitely many discontinuities, each of which is a jump discontinuity (we say that $x$ is a jump discontinuity when $\lim _{z \nearrow x} \tau(z)$ and $\lim _{z \searrow x} \tau(z)$ both exist but are distinct, and one of these values equals $\tau(x)$ ).

If a pre-image selector $\tau$ has $p \geq 1$ discontinuities then the closed set $F=\overline{\tau(\mathbb{T})}$ is called the $p$-flower (or simply the flower) associated to $\tau$. Each of the $p$ connected components of $F$ is a closed interval with non-empty interior, and is called a petal of $F$.

If $F$ is a flower then define

$$
\mathcal{D}_{F}=T(\partial F),
$$

the set of discontinuities of any pre-image selector corresponding to $F$. Examples of flowers and pre-image selectors are shown in Figure 1.

Remark 2.3. (a) A pre-image selector $\tau: \mathbb{T} \rightarrow \tau(\mathbb{T})$ is an inverse map for $\left.T\right|_{\tau(\mathbb{T})}$ (i.e. $T \circ \tau$ is the identity on $\mathbb{T}$, and $\tau \circ T$ is the identity on $\tau(\mathbb{T})$ ). Since an expanding map on $\mathbb{T}$ is not a bijection, a pre-image selector is necessarily discontinuous.

(b) In view of the discussion in $\S 1$, one might envisage relaxing the finiteness assumption in the definitions of pre-image selector and flower. The resulting objects are more complicated than those considered here: for example the next simplest case would allow the set of discontinuities of $\tau$ to be countably infinite. In this case any accumulation point of the set of discontinuities would be a discontinuity but not a jump discontinuity, and already this would lead to certain complications in the proof of any analogue of Theorem 3.6.

(c) We defined flowers above in terms of pre-image selectors. Alternatively we could have defined flowers first, and then associated a (non-unique) pre-image selector. More precisely, if $F \subset \mathbb{T}$ can be written as $F=\bigcup_{j=1}^{p} \overline{J_{j}}$, the closure of a finite union of open intervals $J_{j}$, such that $T(F)=\mathbb{T}$, and $T\left(J_{i}\right) \cap T\left(J_{j}\right)=\emptyset$ for $i \neq j$, then $F$ is the flower associated to some pre-image selector. Indeed there are $2^{p}$ pre-image selectors $\tau$ whose associated flower is $F$. Each such $\tau$ is defined, on the interior of $F$, by $\tau(x)=$ $T^{-1}(x) \cap\left(\bigcup_{i=1}^{p} J_{j}\right)$ for $x \in \bigcup_{i=1}^{p} T\left(J_{j}\right)$; if $x \in T(\partial F)$ then $T^{-1}(x) \cap F=\left\{y, y^{\prime}\right\}$ for some $y \neq y^{\prime}$, and we may define $\tau(x)$ to equal either $y$ or $y^{\prime}$.

(d) Our terminology for flowers differs slightly from that of Brémont [Br1]. What we call a $p$-flower for a degree- $k$ map would, in [Br1], be termed a $k$-flower with $p$ petals.

(e) Every flower carries at least one $T$-invariant probability measure. This is easily proved using the compactness of the flower, and the fact that it contains a pre-image of each point on the circle.

Definition 2.4. Let $T: \mathbb{T} \rightarrow \mathbb{T}$ be an orientation-preserving expanding map, and $\tau$ a preimage selector for $T$. If $x$ is a discontinuity of $\tau$ then it is a jump discontinuity, so there exists $\varepsilon>0$ and $i, j \in\{0, \ldots, k-1\}, i \neq j$, such that

$$
\tau(z)= \begin{cases}T_{i}(z) & \text { for } x-\varepsilon<z<x \\ T_{j}(z) & \text { for } x<z<x+\varepsilon\end{cases}
$$


and $\tau(x)$ equals either $T_{i}(x)$ or $T_{j}(x)$. In this case we say that $x$ is a discontinuity of type $(i, j)$. Note that both the points $T_{i}(x)$ and $T_{j}(x)$ belong to the boundary $\partial F$ of the associated flower $F=\overline{\tau(\mathbb{T})}$, and that no other points in $T^{-1}(x)$ belong to $\partial F$.

For a discontinuity $x$ of $\tau$ we define $y=y(x)$ and $y^{\prime}=y^{\prime}(x)$ by

$$
y(x)=\lim _{z \searrow x} \tau(z), \quad y^{\prime}(x)=\lim _{z \nearrow x} \tau(z),
$$

so in particular $y(x)$ is the left $\dagger$ endpoint of a petal of $F$, and $y^{\prime}(x)$ is the right endpoint of a petal of $F \ddagger$.

Remark 2.5. A pre-image selector for an orientation-preserving Lipschitz expanding map is itself Lipschitz when restricted to any of its finitely many intervals of continuity. Therefore it is differentiable Lebesgue almost everywhere, and its derivative is in $L^{\infty}$, by Lemma 2.1. If the expanding map $T$ satisfies $T^{\prime}(x) \geq K>1$ for Lebesgue almost every $x \in \mathbb{T}$, then the pre-image selector $\tau$ satisfies

$$
0<\tau^{\prime}(x) \leq K^{-1} \quad \text { for Lebesgue almost every } x \in \mathbb{T} .
$$

In particular, the chain rule implies that

$$
\left\|\left(\tau^{n}\right)^{\prime}\right\|_{L^{\infty}} \leq K^{-n} \text { for all } n \geq 1 .
$$

Notation 2.6. Let $\tau: \mathbb{T} \rightarrow \mathbb{T}$ be a pre-image selector, with corresponding flower $F$. Let $x_{1}$ denote that discontinuity of $\tau$ which, with respect to the ordering $<_{0}$, is smaller than all other discontinuities of $\tau \S$. Let $y_{1}$ be the unique pre-image of $x_{1}$ which is a left endpoint of some petal in $F$, and for any $x \in \mathcal{D}_{F}$ define

$$
I_{x}= \begin{cases}{\left[y(x), y^{\prime}(x)\right]} & \text { if } y(x)<y_{1} y^{\prime}(x), \\ {\left[y^{\prime}(x), y(x)\right]} & \text { if } y^{\prime}(x)<y_{1} y(x) .\end{cases}
$$

Define

$$
\mathcal{A}=\left\{x \in \mathcal{D}_{F}: I_{x}=\left[y(x), y^{\prime}(x)\right]\right\}, \quad \mathcal{A}^{\prime}=\mathcal{D}_{F} \backslash \mathcal{A} .
$$

Notation 2.7. For a subset $G \subset \mathbb{T}$, let $\chi(G)$ denote its characteristic function.

The following Lemma 2.8 will be a very useful tool in $\S 3$. For maps $T$ of degree $k>2$, the combinatorics involved in describing which pre-image of a point lies in a flower $F$ is significantly more complicated than in the degree-two case. Lemma 2.8 , which is used in the proof of both Theorems 3.6 and 3.8, allows us to efficiently sidestep these complications.

LEMMA 2.8. For any flower $F$, we have

$$
\chi(F)=\sum_{x \in \mathcal{A}} \chi\left(I_{x}\right)-\sum_{x \in \mathcal{A}^{\prime}} \chi\left(I_{x}\right) .
$$

$\dagger$ If $T$ were orientation-reversing then $y(x)$ would be a right endpoint, and $y^{\prime}(x)$ a left endpoint.

$\ddagger$ In general $y(x)$ and $y^{\prime}(x)$ may, or may not, be endpoints of the same petal.

$\S$ This choice of $x_{1}$ is for definiteness; in fact it is possible to fix $x_{1}$ to be an arbitrary discontinuity of $\tau$. 
Proof. Define $g: \mathbb{T} \rightarrow \mathbb{R}$ by

$$
g=\sum_{x \in \mathcal{A}} \chi\left(I_{x}\right)-\sum_{x \in \mathcal{A}^{\prime}} \chi\left(I_{x}\right) .
$$

The function $g$ is upper semi-continuous and piecewise constant: its discontinuities are at the points $y(x)$ and $y^{\prime}(x)$, for $x \in \mathcal{D}_{F}$.

We claim that, at every left endpoint $y$ of a petal of $F$, the function $g$ increases by 1 , in the sense that $g(y)=1+\lim _{z \nearrow y} g(z)$. To see this, recall that every such left endpoint is of the form $y=y(x)$ for some $x \in \mathcal{D}_{F}$. If $x \in \mathcal{A}$ then $\chi\left(I_{x}\right)=\chi\left(\left[y(x), y^{\prime}(x)\right]\right)$ increases by 1 at the point $y(x)$, while $g-\chi\left(I_{x}\right)$ is locally constant at $y(x)$, so $g=\chi\left(I_{x}\right)+\left(g-\chi\left(I_{x}\right)\right)$ increases by 1 at $y(x)$. If $x \in \mathcal{A}^{\prime}$ then $\chi\left(I_{x}\right)=\chi\left(\left[y^{\prime}(x), y(x)\right]\right)$ decreases by 1 at the point $y(x)$, while $g+\chi\left(I_{x}\right)$ is locally constant at $y(x)$, so $g=-\chi\left(I_{x}\right)+\left(g+\chi\left(I_{x}\right)\right)$ increases by 1 at $y(x)$.

Similarly, we can show that at every right endpoint $y^{\prime}$ of a petal of $F$, the function $g$ decreases by 1 , in the sense that $g\left(y^{\prime}\right)=1+\lim _{z \searrow y} g(z)$.

Now $y_{1}$ is the smallest point in the ordered set $\left(\mathbb{T},<_{y_{1}}\right)$, so $y_{1} \in I_{x_{1}}$, but $y_{1} \notin I_{x}$ for $x \in \mathcal{D}_{F} \backslash\left\{x_{1}\right\}$. Therefore $g\left(y_{1}\right)=1$. With respect to $<_{y_{1}}$, the left and right endpoints of petals of $F$ alternate around the circle, beginning with a right endpoint after $y_{1}$. Therefore $g$ takes the value 1 between left and right endpoints of petals of $F$, and takes the value 0 between right and left endpoints of petals of $F$. That is, $g=\chi(F)$.

\section{Lipschitz flattening}

Recall that $\mathcal{M}_{T}$ denotes the set of $T$-invariant Borel probability measures. A continuous function $g: \mathbb{T} \rightarrow \mathbb{R}$ is called a weak coboundary if $\int g d \mu=0$ for every $\mu \in \mathcal{M}_{T}$, and a coboundary if $g=\varphi-\varphi \circ T$ for some continuous $\varphi: \mathbb{T} \rightarrow \mathbb{R}$. Clearly every coboundary is a weak coboundary, but the converse is not true (see e.g. [BJ]). However, if $g$ is Lipschitz, then it is a coboundary if and only if it is a weak coboundary, and if so then there is a Lipschitz $\varphi$ (which is unique up to an additive constant) such that $g=\varphi-\varphi \circ T$ (see [Liv]).

Definition 3.1. Let $T: \mathbb{T} \rightarrow \mathbb{T}$ be an expanding map, and $F$ a corresponding flower. A continuous function $f: \mathbb{T} \rightarrow \mathbb{R}$ is said to be flat on $F$ if the restriction $\left.f\right|_{F}$ is a constant function.

We say that $f$ can be continuously flattened on $F$ if there exists a weak coboundary $g: \mathbb{T} \rightarrow \mathbb{R}$ such that $f+g$ is flat on $F$.

We say that $f$ can be Lipschitz flattened on $F$ if there exists a Lipschitz coboundary $g: \mathbb{T} \rightarrow \mathbb{R}$ such that $f+g$ is flat on $F$ (or, equivalently, there exists a Lipschitz function $\varphi: \mathbb{T} \rightarrow \mathbb{R}$ such that $f+\varphi-\varphi \circ T$ is flat on $F$ ).

Remark 3.2. If $f$ can be continuously flattened on $F$ then the constant function $\left.(f+g)\right|_{F}$ is identically equal to $\int f d \mu$, where $\mu$ is any $T$-invariant probability measure carried by $F$. If there are several such measures then this provides an obstruction to being able to continuously flatten a given function on $F$ : its integral must be the same with respect to each measure. For example if $T(x)=2 x(\bmod 1)$, and $F=\left[-\frac{1}{12}, \frac{1}{12}\right] \cup\left[\frac{1}{4}, \frac{5}{12}\right] \cup$ $\left[\frac{7}{12}, \frac{3}{4}\right]$, then $F$ contains both the fixed point 0 and the period-two orbit $\{1 / 3,2 / 3\}$, so 


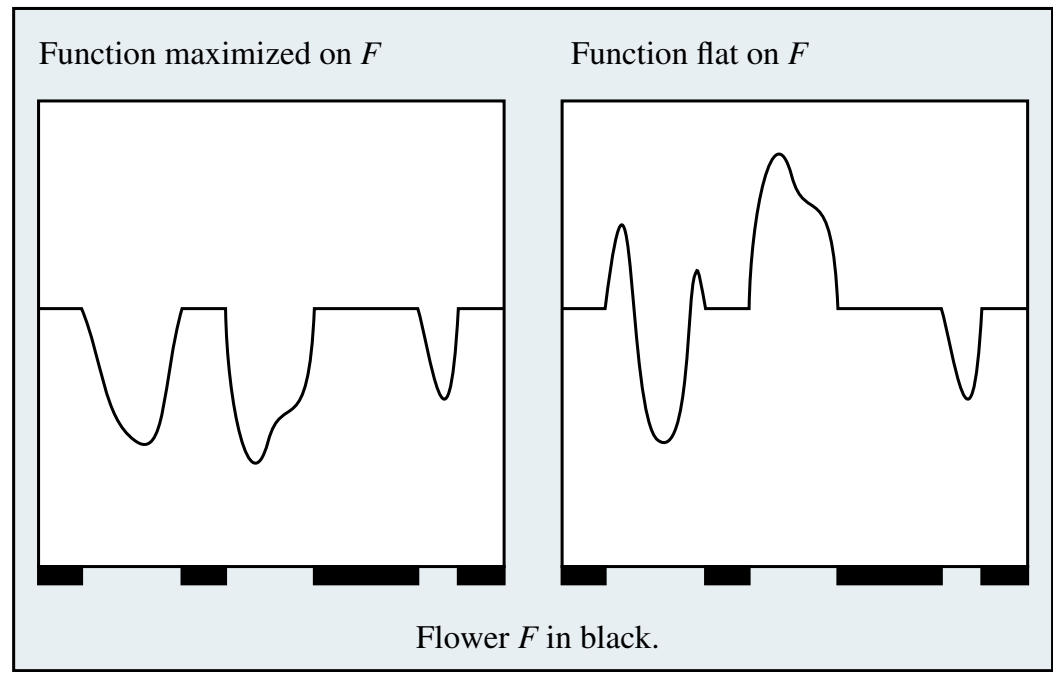

FIgURE 2. Two functions flat on the flower $F$ (a 3-flower for the doubling map $T_{2}(x)=2 x$ (mod 1)). The first function has the additional property that it attains its global maximum precisely on $F$, so in particular is in normal form (cf. Definition 4.6); its maximizing measures are therefore precisely those invariant measures carried by $F$.

a necessary condition for continuously flattening $f$ on $F$ is that $f(0)=\frac{1}{2}(f(1 / 3)+$ $f(2 / 3))$.

Notation 3.3. Let Lip denote the vector space of all real-valued Lipschitz functions on $\mathbb{T}$. For a flower $F \subset \mathbb{T}$, define

$$
\operatorname{Lip}_{F}=\{f \in \operatorname{Lip}: f \text { can be Lipschitz flattened on } F\} .
$$

Using the various definitions and notation introduced so far, it is now possible to reiterate the main results of this paper, in more detail than was possible in $\S 1$. The following is proved as Theorems 3.6 and 3.8.

THEOREM. For any $p$-flower $F$, the set $\operatorname{Lip}_{F}$ is a codimension- $p$ subspace of Lip. Indeed there exist p measures on $\mathbb{T}$, each absolutely continuous with respect to Lebesgue measure, such that $\operatorname{Lip}_{F}$ consists precisely of those Lipschitz functions whose derivative has zero integral with respect to each of these measures.

The Radon-Nikodym derivative of each of the $p$ measures in the above theorem is a certain infinite sum of characteristic functions of intervals (explicit expressions are given in Definition 3.4 below, see (5)). These Radon-Nikodym derivatives can be rapidly approximated (e.g. by truncation of (5)), hence so can the kernels of the corresponding measures (considered as functionals on Lip), and therefore so can the members of $\operatorname{Lip}_{F}$.

As mentioned in $\S 1$, if a function $f$ can be Lipschitz flattened on $F$, then the $T$-invariant measures carried by $F$ may, in certain circumstances, be good candidates for $f$-maximizing measures. The relation between flattening and maximizing is particularly close when $F$ is a 1-flower: for any Lipschitz function $f: \mathbb{T} \rightarrow \mathbb{R}$ we have the following result, which is proved later as Theorem 4.9. 
THEOREM. If the $f$-maximizing measure $S$ is carried by some 1 -flower $F$, then $f$ can be Lipschitz flattened on some 1-flower $F^{\prime}$ which carries $S$.

It should be noted that $F=F^{\prime}$ for non-atomic $S$, while if $S$ is atomic then $F^{\prime}$ need not equal $F$. The importance of the above theorem stems from the fact that invariant measures carried by 1-flowers (so-called Sturmian measures, cf. $\S 1$ and Lemma 4.4) tend to arise as $f$-maximizing measures for sufficiently simple functions $f$ (cf. [ADJR, Bou1, Jen1, Jen3, Jen4]). The theorem implies that, for such $f$, the problem of precisely identifying the $f$-maximizing measure is reduced to determining those 1-flowers on which $f$ can be flattened, a problem which is computationally accessible.

Definition 3.4. Let $\tau: \mathbb{T} \rightarrow \mathbb{T}$ be a pre-image selector, with corresponding flower $F$. For each $x \in \mathcal{D}_{F}$, define $e_{x}: \mathbb{T} \rightarrow \mathbb{R}$ by

$$
e_{x}=\sum_{n=0}^{\infty} \chi\left(\tau^{n} I_{x}\right) .
$$

Note that $e_{x} \in L^{1}$, because the Lebesgue measure of $\tau^{n} I_{x}$ decreases exponentially with $n$, by (4).

Remark 3.5. If $F$ is a 1 -flower then a corresponding pre-image selector has just one point of discontinuity $x$, and $F=\left[y, y^{\prime}\right]$, where $y^{\prime}=T_{i}(x), y=T_{i+1}(x)$ for some $0 \leq i \leq k-2$ (or $y^{\prime}=T_{k-1}(x), y=T_{0}(x)$ ). In this case the function $e_{x}$ will be denoted by $e_{F}$, and can be interpreted as the escape time function for $F$,

$$
e_{x}(t)=e_{F}(t)=\inf \left\{n \geq 0: T^{n}(t) \notin F\right\} .
$$

If $f$ is itself Lipschitz then the following result, which generalizes [Bou1, Proposition, p. 503], gives necessary and sufficient conditions for being able to Lipschitz flatten $f$ on flowers.

THEOREM 3.6. Let $T: \mathbb{T} \rightarrow \mathbb{T}$ be an orientation-preserving Lipschitz expanding map. Let $F \subset \mathbb{T}$ be a flower, and $\tau$ a corresponding pre-image selector. If $f: \mathbb{T} \rightarrow \mathbb{R}$ is Lipschitz, then the following are equivalent:

(a) $f$ can be Lipschitz flattened on $F$;

(b) for each discontinuity $x$ of $\tau$,

$$
\int \sum_{I_{x}}^{\infty}\left(f \circ \tau^{n}\right)^{\prime}=0
$$

(c) for each discontinuity $x$ of $\tau$,

$$
\int e_{x} f^{\prime}=0
$$

Proof. (a) $\Rightarrow$ (b) If $f$ can be Lipschitz flattened on $F$ then there is a Lipschitz function $\varphi: \mathbb{T} \rightarrow \mathbb{R}$, and a constant $c \in \mathbb{R}$, such that $f+\varphi-\varphi \circ T=c$ on $F$. In particular, we have $(f+\varphi-\varphi \circ T)(z)=c$ for all $z \in \tau(\mathbb{T})$. Writing $z=\tau(x)$, and recalling that $\tau: \mathbb{T} \rightarrow \tau(\mathbb{T})$ is a bijection such that $T \circ \tau$ is the identity on $\mathbb{T}$, we derive

$$
(f+\varphi) \circ \tau(x)=\varphi(x)+c \text { for all } x \in \mathbb{T} .
$$


Since $\varphi$ is continuous on $\mathbb{T}$, equation (6) implies that $(f+\varphi) \circ \tau$ is also continuous on $\mathbb{T}$. In particular, $(f+\varphi) \circ \tau$ is continuous at each discontinuity $x$ of $\tau$. This discontinuity is of type $(i, j)$ for some $1 \leq i, j \leq p$, so there exists $\varepsilon>0$ such that $\tau(z)=T_{i}(z)$ for $z \in(x-\varepsilon, x)$ and $\tau(z)=T_{j}(z)$ for $z \in(x, x+\varepsilon)$. So $T^{-1}(x) \cap F=\left\{y, y^{\prime}\right\}$, where $y^{\prime}=T_{i}(x)$ is the right endpoint of a petal in $F$, and $y=T_{j}(x)$ is the left endpoint of a petal in $F$. Therefore

$$
\begin{aligned}
(f+\varphi)(y) & =(f+\varphi)\left(T_{j}(x)\right)=\lim _{z \nearrow x}(f+\varphi)\left(T_{j}(z)\right) \\
& =\lim _{z \searrow x}(f+\varphi)\left(T_{i}(z)\right)=(f+\varphi)\left(T_{i}(x)\right)=(f+\varphi)\left(y^{\prime}\right) .
\end{aligned}
$$

Now $f+\varphi$ is Lipschitz, hence absolutely continuous, so by the fundamental theorem of calculus,

$$
\begin{aligned}
\int_{y}^{y^{\prime}}(f+\varphi)^{\prime} & =(f+\varphi)\left(y^{\prime}\right)-(f+\varphi)(y)=0 \\
& =(f+\varphi)(y)-(f+\varphi)\left(y^{\prime}\right)=\int_{y^{\prime}}^{y}(f+\varphi)^{\prime}
\end{aligned}
$$

In particular, we have

$$
\int_{I_{x}}(f+\varphi)^{\prime}=0 .
$$

Now iteration of (6) gives $\varphi=-m c+\sum_{n=1}^{m} f \circ \tau^{n}+\varphi \circ \tau^{m}$, and differentiation yields $\varphi^{\prime}=\sum_{n=1}^{m}\left(f \circ \tau^{n}\right)^{\prime}+\left(\varphi \circ \tau^{m}\right)^{\prime}$ in $L^{\infty}$. But $\left(\varphi \circ \tau^{m}\right)^{\prime}=\varphi^{\prime} \circ \tau^{m} \cdot\left(\tau^{m}\right)^{\prime} \rightarrow 0$ in $L^{\infty}$, because $\left\|\left(\tau^{m}\right)^{\prime}\right\|_{L^{\infty}} \rightarrow 0$ as $m \rightarrow \infty$ by (4), so

$$
\varphi^{\prime}=\sum_{n=1}^{\infty}\left(f \circ \tau^{n}\right)^{\prime} \quad \text { in } L^{\infty} .
$$

Substituting (8) into (7) gives $\int_{I_{x}} \sum_{n=0}^{\infty}\left(f \circ \tau^{n}\right)^{\prime}=0$, as required.

(a) $\Leftarrow$ (b) By Lemma 2.8, the characteristic function $\chi(F)$ can be expressed as a linear combination of the characteristic functions $\chi\left(I_{x}\right)$, for $x \in \mathcal{D}_{F}$. So the integral of a function over $F$ is a linear combination of its integrals over the intervals $I_{x}$, for $x \in \mathcal{D}_{F}$. In particular, condition (b) implies that

$$
\int_{F} \sum_{n=0}^{\infty}\left(f \circ \tau^{n}\right)^{\prime}=0,
$$

which is equivalent, by change of variable, to

$$
\int \sum_{n=1}^{\infty}\left(f \circ \tau^{n}\right)^{\prime}=0 .
$$

The function $x \mapsto \sum_{n=1}^{\infty}\left(f \circ \tau^{n}\right)^{\prime}(x)$, defined Lebesgue almost everywhere, is $L^{\infty}$ : each summand $x \mapsto\left(f \circ \tau^{n}\right)^{\prime}(x)$ is in $L^{\infty}$ because $f$ is Lipschitz and $\tau$ is piecewise Lipschitz, and the sum converges in $L^{\infty}$ since $\left(f \circ \tau^{n}\right)^{\prime}(x)=f^{\prime}\left(\tau^{n} x\right)\left(\tau^{n}\right)^{\prime}(x)$, and $\left\|\left(\tau^{n}\right)^{\prime}\right\|_{L^{\infty}} \leq$ $K^{-n}$ by (4). So by (9) the function $x \mapsto \sum_{n=1}^{\infty}\left(f \circ \tau^{n}\right)^{\prime}(x)$ lies in $L_{0}^{\infty}$, and by Lemma 2.1 it is the derivative of some Lipschitz continuous function $\varphi: \mathbb{T} \rightarrow \mathbb{R}$. Now

$$
(\varphi \circ T-(f+\varphi))^{\prime}=0 \quad \text { Lebesgue almost everywhere on } F,
$$


since $\tau \circ T$ is Lebesgue almost everywhere equal to the identity function on $F$. But $\varphi \circ T-(f+\varphi)$ is continuous, so by (10) its restriction to $F$ is a constant function.

(b) $\Leftrightarrow$ (c) If $x$ is a discontinuity of $\tau$, then

$$
\begin{aligned}
\int_{I_{x}} \sum_{n=0}^{\infty}\left(f \circ \tau^{n}\right)^{\prime} & =\sum_{n=0}^{\infty} \int_{I_{x}}\left(f \circ \tau^{n}\right)^{\prime}=\sum_{n=0}^{\infty} \int_{\tau^{n} I_{x}} f^{\prime} \\
& =\sum_{n=0}^{\infty} \int \chi\left(\tau^{n} I_{x}\right) f^{\prime}=\int \sum_{n=0}^{\infty} \chi\left(\tau^{n} I_{x}\right) f^{\prime}=\int e_{x} f^{\prime}
\end{aligned}
$$

and the equivalence of (b) and (c) follows.

Remark 3.7. (a) A further condition equivalent to those of Theorem 3.6 is that, for all $x \in \mathcal{D}_{F}$,

$$
\int_{y}^{y^{\prime}} \sum_{n=0}^{\infty}\left(f \circ \tau^{n}\right)^{\prime}=0=\int_{y^{\prime}}^{y} \sum_{n=0}^{\infty}\left(f \circ \tau^{n}\right)^{\prime} .
$$

Clearly (11) implies condition (b) of Theorem 3.6, while the fact that (11) is implied by condition (a) of Theorem 3.6 was essentially established during the proof that (a) $\Rightarrow$ (b).

If, for $x \in \mathcal{D}_{F}$, we define $d_{x}: \mathbb{T} \rightarrow \mathbb{R}$ by

$$
d_{x}=\sum_{n=0}^{\infty} \chi\left(\tau^{n} J_{x}\right)
$$

where

$$
J_{x}= \begin{cases}{\left[y^{\prime}(x), y(x)\right]} & \text { if } y(x)<_{y_{1}} y^{\prime}(x) \\ {\left[y(x), y^{\prime}(x)\right]} & \text { if } y^{\prime}(x)<_{y_{1}} y(x)\end{cases}
$$

then (11) becomes

$$
\int_{I_{x}} \sum_{n=0}^{\infty}\left(f \circ \tau^{n}\right)^{\prime}=0=\int_{J_{x}} \sum_{n=0}^{\infty}\left(f \circ \tau^{n}\right)^{\prime} \quad \text { for all } x \in \mathcal{D}_{F},
$$

from which it easily follows that the condition

$$
\int d_{x} f^{\prime}=0 \quad \text { for all } x \in \mathcal{D}_{F}
$$

is also equivalent to those of Theorem 3.6.

(b) The proof of Theorem 3.6 implies that, if the Lipschitz function $f$ can be Lipschitz flattened on the flower $F$, then there is a unique Lipschitz coboundary $g$ such that $\left.(f+g)\right|_{F}$ is constant. This is because the corresponding pre-image selector $\tau$ and all of its iterates $\tau^{n}$ are uniquely defined on a set of full Lebesgue measure, and if we write $g=$ $\varphi-\varphi \circ T$ then $\varphi$ is uniquely defined, up to an additive constant, by $\varphi^{\prime}=\sum_{n=1}^{\infty}\left(f \circ \tau^{n}\right)^{\prime}$ (see (8)).

THEOREM 3.8. Let $T: \mathbb{T} \rightarrow \mathbb{T}$ be an orientation-preserving Lipschitz expanding map. If $F \subset \mathbb{T}$ is a $p$-flower then $\operatorname{Lip}_{F}$ is a codimension-p subspace of Lip. 
Proof. Let $x_{1}, \ldots, x_{p}$ denote the discontinuities of a pre-image selector $\tau$ for $F$. For $1 \leq j \leq p$, the function $e_{x_{j}}$ will be denoted simply by $e_{j}$. Define the linear functional $L_{F, j}: \operatorname{Lip} \rightarrow \mathbb{R}$ by $L_{F, j}(f)=\int e_{j} f^{\prime}$. By Theorem 3.6,

$$
\operatorname{Lip}_{F}=\left\{f \in \operatorname{Lip}: L_{F, j}(f)=0 \text { for } 1 \leq j \leq p\right\},
$$

so $\operatorname{Lip}_{F}$ has codimension $p$ in Lip if and only if the $p$ functionals $\left\{L_{F, j}\right\}_{j=1}^{p}$ are linearly independent. This linear independence is equivalent to the fact that

$$
\int\left(\sum_{j=1}^{p} \alpha_{j} e_{j}\right) f^{\prime}=0 \text { for all } f \in \operatorname{Lip} \Rightarrow\left(\alpha_{1}, \ldots, \alpha_{p}\right)=(0, \ldots, 0),
$$

and, since $f^{\prime} \in L_{0}^{\infty}$ for all $f \in \operatorname{Lip}$, this is equivalent to the fact that

$$
\sum_{j=1}^{p} \alpha_{j} e_{j} \text { is a constant function } \Rightarrow\left(\alpha_{1}, \ldots, \alpha_{p}\right)=(0, \ldots, 0) \text {. }
$$

So suppose that $\sum_{j=1}^{p} \alpha_{j} e_{j}$ is a constant function. The strategy for showing that $\alpha_{j}=0$ for $1 \leq j \leq p$ will be to consider the values of the functions $e_{j}$ on the $p$ connected components of the complement of $F$, and on the image under $\tau$ of a particular one of these components.

For $1 \leq j \leq p$, define the intervals $I_{j}=I_{x_{j}}$ as in Notation 2.6, with reference to the counterclockwise ordering $<_{y_{1}}$ (i.e. the smallest element is $y_{1}$, the unique pre-image of $x_{1}$ which is a left endpoint of some petal in $F$ ). Denote the $p$ petals of $F$ by $P_{1}, \ldots, P_{p}$, ordered counterclockwise and such that $P_{1}$ is the petal whose left endpoint is $y_{1}$. Let $Q_{1}, \ldots, Q_{p}$ denote the $p$ connected components of $\mathbb{T} \backslash F$, ordered counterclockwise and such that $Q_{1}$ is the component whose left endpoint is the right endpoint of $P_{1}$.

First of all note that $Q_{p}$ does not intersect any of the intervals $I_{j}$. Moreover $Q_{p}$ does not intersect $F=\overline{\tau(\mathbb{T})}$, hence does not intersect any of the sets $\tau^{n}\left(I_{j}\right)$ for $1 \leq j \leq p, n \geq 1$. Therefore each function $e_{j}=\sum_{n \geq 0} \chi\left(\tau^{n} I_{j}\right)$ is identically zero on the interval $Q_{p}$. So if $\sum_{j=1}^{p} \alpha_{j} e_{j}$ is a constant function on $\mathbb{T}$, then this constant must be zero.

It remains to show that if the function $\sum_{j=1}^{p} \alpha_{j} e_{j}$ is identically zero then $\alpha_{j}=0$ for $1 \leq j \leq p$. For this we will consider the function on the other $p-1$ components $Q_{1}, \ldots, Q_{p-1}$ of the complement of $F$, as well as on the set $\tau\left(Q_{p}\right)$.

First consider the restriction of the functions $e_{j}$ to the remaining $p-1$ components $Q_{1}, \ldots, Q_{p-1}$ of the complement of $F$. Let

$$
\mathcal{B}=\left\{j: x_{j} \in \mathcal{A}\right\}, \quad \mathcal{B}^{\prime}=\left\{j: x_{j} \in \mathcal{A}^{\prime}\right\},
$$

and for each $1 \leq i \leq p$ define

$$
\mathcal{B}_{i}=\left\{j \in \mathcal{B}: Q_{i} \subset I_{j}\right\}, \quad \mathcal{B}_{i}^{\prime}=\left\{j \in \mathcal{B}^{\prime}: Q_{i} \subset I_{j}\right\} .
$$

Note that, for any $1 \leq i, j \leq p, Q_{i}$ either is a subset of $I_{j}$ or is disjoint from $I_{j}$, and

$$
\left.e_{j}\right|_{Q_{i}} \equiv \begin{cases}0 & \text { when } Q_{i} \cap I_{j}=\emptyset \\ 1 & \text { when } Q_{i} \subset I_{j}\end{cases}
$$


The restriction to $Q_{i}$ of the equation $\sum_{j=1}^{p} \alpha_{j} e_{j}=0$ therefore yields

$$
\sum_{j \in \mathcal{B}_{i} \cup \mathcal{B}_{i}^{\prime}} \alpha_{j}=0 \quad \text { for all } 1 \leq i \leq p-1 .
$$

It will be convenient to consider the case $i=1$ of (13), together with the system of equations obtained by subtracting the $(i-1)$ th equation (13) from the $i$ th equation (13) for $2 \leq i \leq p-1$. Since $Q_{i}$ is disjoint from $F$, Lemma 2.8 implies that

$$
\sum_{j \in \mathcal{B}} \chi\left(I_{j}\right)=\sum_{j \in \mathcal{B}^{\prime}} \chi\left(I_{j}\right) \quad \text { on } Q_{i}, \text { for all } 1 \leq i \leq p .
$$

For each $2 \leq i \leq p-1$, if we write $P_{i}=\left[y_{k_{i}}, y_{l_{i}}^{\prime}\right]$ then, in view of (14), subtracting the $(i-1)$ th equation (13) from the $i$ th equation (13) yields

$$
\alpha_{k_{i}}=\alpha_{l_{i}} \text { if } k_{i}, l_{i} \in \mathcal{B} \text { or } k_{i}, l_{i} \in \mathcal{B}^{\prime},
$$

and

$$
\alpha_{k_{i}}=-\alpha_{l_{i}} \quad \text { if } k_{i} \in \mathcal{B}, l_{i} \in \mathcal{B}^{\prime} \text { or } k_{i} \in \mathcal{B}^{\prime}, l_{i} \in \mathcal{B} .
$$

Now consider $\{1, \ldots, p\}$ as the vertex set for an undirected graph $\Gamma$, where there is an edge between $k$ and $l$ if and only if some petal of $F$ is equal to $\left[y_{k}, y_{l}^{\prime}\right]$. If we can prove that $\Gamma$ is connected then the equation (13) with $i=1$, together with the equations (15) and (16) for $2 \leq i \leq p-1$, imply that

$$
\left(\alpha_{1}, \ldots, \alpha_{p}\right)=t\left(\beta_{1}, \ldots, \beta_{p}\right)
$$

for some $t \in \mathbb{R}$, where

$$
\beta_{j}= \begin{cases}1 & \text { if } j \in \mathcal{B}, \\ -1 & \text { if } j \in \mathcal{B}^{\prime}\end{cases}
$$

To prove that $\Gamma$ is connected, suppose for a contradiction that it is not, and let $\mathcal{C} \subsetneq\{1, \ldots, p\}$ be the vertex set corresponding to some connected component of $\Gamma$. Let $\mathcal{D}=\left\{y_{k} \in \mathbb{T}: k \in \mathcal{C}\right\} \cup\left\{y_{k}^{\prime} \in \mathbb{T}: k \in \mathcal{C}\right\}$, and let $E \subset F$ denote the union of those petals of $F$ whose endpoints lie in $\mathcal{D}$. Now at least one petal of $F$ is disjoint from $E$, and every petal in $E$ has positive length, so since $T$ is Lipschitz,

$$
0<\operatorname{Leb}(T(E))<1 .
$$

For each $1 \leq j \leq p$, Lebesgue almost every point in $\mathbb{T}$ has the same number of pre-images (under $T$ ) lying in $I_{j}$. But

$$
\chi(E)=\sum_{j \in \mathcal{C} \cap \mathcal{B}} \chi\left(I_{j}\right)-\sum_{j \in \mathcal{C} \cap \mathcal{B}^{\prime}} \chi\left(I_{j}\right),
$$

so Lebesgue almost every point in $\mathbb{T}$ has the same number of pre-images (under $T$ ) lying in $E$, which contradicts (18). So $\Gamma$ is in fact connected, and therefore (17) holds.

It remains to show that in fact $t=0$ in (17). For this, we shall consider the values of the functions $e_{j}$ on the set $\tau\left(Q_{p}\right)$. More precisely, if $P_{i}$ is a petal of $F$ whose interior has non-empty intersection with $\tau\left(Q_{p}\right)$ then we shall consider the values of the functions $e_{j}$ on 
$\operatorname{int}\left(P_{i}\right) \cap \tau\left(Q_{p}\right)$. Note that each $e_{j}$ is identically equal to either 0 or 1 on $\operatorname{int}\left(P_{i}\right) \cap \tau\left(Q_{p}\right)$; it is identically equal to 1 if and only if $I_{j}$ contains $P_{i}$.

Let us write $P_{i}=\left[y_{k}, y_{l}^{\prime}\right]$. If $j \in\{1, \ldots, p\} \backslash\{l\}$ then $Q_{i} \subset I_{j}$ if and only if $\operatorname{int}\left(P_{i}\right) \cap$ $\tau\left(Q_{p}\right) \subset I_{j}$, so the constant value of $e_{j}$ on $Q_{i}$ is the same as its constant value on $\operatorname{int}\left(P_{i}\right) \cap \tau\left(Q_{p}\right)$. If $l \in \mathcal{B}$ then $Q_{i} \cap I_{l}=\emptyset$ and $P_{i} \subset I_{l}$, hence $\operatorname{int}\left(P_{i}\right) \cap \tau\left(Q_{p}\right) \subset I_{l}$, so $\left.e_{j}\right|_{Q_{i}} \equiv 0$ while $e_{j} \mid \operatorname{int}\left(P_{i}\right) \cap \tau\left(Q_{p}\right) \equiv 1$. If $l \in \mathcal{B}^{\prime}$ then $Q_{i} \subset I_{l}$ and $\operatorname{int}\left(P_{i}\right) \cap I_{l}=\emptyset$, hence $\left(\operatorname{int}\left(P_{i}\right) \cap \tau\left(Q_{p}\right)\right) \cap I_{l}=\emptyset$, so $\left.e_{j}\right|_{Q_{i}} \equiv 1$ while $\left.e_{j}\right|_{\operatorname{int}\left(P_{i}\right) \cap \tau\left(Q_{p}\right)} \equiv 0$. Therefore, subtracting the restriction of the equation $\sum_{j=1}^{p} \alpha_{j} e_{j}=0$ to $\operatorname{int}\left(P_{i}\right) \cap \tau\left(Q_{p}\right)$ from the restriction of the same equation to $Q_{i}$ yields

$$
\alpha_{l}=0,
$$

and from (17) we deduce that $\left(\alpha_{1}, \ldots, \alpha_{p}\right)=(0, \ldots, 0)$, as required.

\section{Flattening on 1-flowers}

Let $T: \mathbb{T} \rightarrow \mathbb{T}$ be an orientation-preserving Lipschitz expanding map. Let $F$ be a 1-flower for $T$, and let $e_{F}$ denote the corresponding escape time function, defined (cf. Remark 3.5) by

$$
e_{F}=\sum_{n \geq 0} \chi\left(\tau_{F}^{n} F\right)
$$

To prove Theorem 4.9 below, it will be useful to know that the $L^{1}$ function $e_{F}$ varies continuously with the 1-flower $F$. The set of all such 1-flowers forms a one-parameter family $\left(F_{\gamma}\right)_{\gamma \in \mathbb{T}}$. Since every 1 -flower $F_{\gamma}$ is in particular a closed proper sub-interval of $\mathbb{T}$, with endpoints $a(\gamma)$ and $b(\gamma)$, say, we may write $F_{\gamma}=[a(\gamma), b(\gamma)]$, where both $\gamma \mapsto a(\gamma)$ and $\gamma \mapsto b(\gamma)$ are degree-one homeomorphisms of $\mathbb{T}$.

Proposition 4.1. The map $\mathbb{T} \rightarrow L^{1}$, defined by $\gamma \mapsto e_{F_{\gamma}}$, is continuous.

Proof. Given any $\eta>0$, we shall show that there exists $\xi>0$ such that if $d(\gamma, \delta)<\xi$ then $\int\left|e_{F_{\gamma}}-e_{F_{\delta}}\right|<\eta$.

Let $|G|$ denote the Lebesgue measure of a measurable subset $G \subset \mathbb{T}$. If $K>1$ is the expanding constant as in (1) then

$$
\left|\tau_{\gamma}^{n} F_{\gamma}\right| \leq K^{-n}\left|F_{\gamma}\right|<K^{-n}
$$

and

$$
\left|\tau_{\delta}^{n} F_{\delta}\right| \leq K^{-n}\left|F_{\delta}\right|<K^{-n}
$$

by (4), so, for all $N \in \mathbb{N}$,

$$
\begin{aligned}
\int\left|\sum_{n>N} \chi\left(\tau_{\gamma}^{n} F_{\gamma}\right)-\sum_{n>N} \chi\left(\tau_{\delta}^{n} F_{\delta}\right)\right| & \leq \sum_{n>N} \int \chi\left(\tau_{\gamma}^{n} F_{\gamma}\right)+\chi\left(\tau_{\delta}^{n} F_{\delta}\right) \\
& <2 \sum_{n>N} K^{-n}=\frac{2 K^{-(N+1)}}{1-K^{-1}} .
\end{aligned}
$$

In particular, we may choose $N$ sufficiently large so that

$$
\int\left|\sum_{n>N} \chi\left(\tau_{\gamma}^{n} F_{\gamma}\right)-\sum_{n>N} \chi\left(\tau_{\delta}^{n} F_{\delta}\right)\right|<\eta / 2 .
$$


It remains to show that we can find $\xi>0$ such that if $d(\gamma, \delta)<\xi$ then

$$
\int\left|\sum_{n=0}^{N} \chi\left(\tau_{\gamma}^{n} F_{\gamma}\right)-\sum_{n=0}^{N} \chi\left(\tau_{\delta}^{n} F_{\delta}\right)\right|<\eta / 2,
$$

or, in other words,

$$
\sum_{n=0}^{N}\left|\tau_{\gamma}^{n} F_{\gamma} \triangle \tau_{\delta}^{n} F_{\delta}\right|<\eta / 2
$$

where $\triangle$ denotes symmetric difference.

The two ingredients for proving (19) are that, if $\gamma$ and $\delta$ are close, then firstly $F_{\gamma} \triangle F_{\delta}$ is small, and secondly the functions $\tau_{\gamma}$ and $\tau_{\delta}$ agree except on a small set. We now make this precise.

The continuity of $\gamma \mapsto a(\gamma)$ and $\gamma \mapsto b(\gamma)$ means that for any $\varepsilon_{0}>0$ we may choose $\xi>0$ such that if $d(\gamma, \delta)<\xi$ then $d(a(\gamma), a(\delta))<\varepsilon_{0}$ and $d(b(\gamma), b(\delta))<\varepsilon_{0}$. In particular,

$$
\left|F_{\gamma} \triangle F_{\delta}\right|<2 \varepsilon_{0} \text {. }
$$

Let $d(\gamma, \delta)$ be small enough so that $F_{\gamma}$ and $F_{\delta}$ intersect. The maps $\tau_{\gamma}$ and $\tau_{\delta}$ are identical except on the interval between their respective points of discontinuity $T(a(\gamma))=$ $T(b(\gamma))$ and $T(a(\delta))=T(b(\delta))$. If we denote this interval by $A=A(\gamma, \delta)$, then

$$
|A|=d(T(a(\gamma)), T(a(\delta))) \leq C d(a(\gamma), a(\delta))<C \varepsilon_{0},
$$

where $C>1$ is the Lipschitz constant for $T$ (cf. (2)).

Now $\tau_{\gamma}^{n} F_{\gamma} \triangle \tau_{\delta}^{n} F_{\delta}$ is contained in

$$
\begin{aligned}
& \tau_{\gamma}\left(\tau_{\gamma}^{n-1} F_{\gamma}\right.\left.\cap \tau_{\delta}^{n-1} F_{\delta} \cap A\right) \cup \tau_{\delta}\left(\tau_{\gamma}^{n-1} F_{\gamma} \cap \tau_{\delta}^{n-1} F_{\delta} \cap A\right) \\
& \cup \tau_{\gamma}\left(\tau_{\gamma}^{n-1} F_{\gamma} \backslash \tau_{\delta}^{n-1} F_{\delta}\right) \cup \tau_{\delta}\left(\tau_{\delta}^{n-1} F_{\delta} \backslash \tau_{\gamma}^{n-1} F_{\gamma}\right),
\end{aligned}
$$

which is itself a subset of

$$
\tau_{\gamma}(A) \cup \tau_{\delta}(A) \cup \tau_{\gamma}\left(\tau_{\gamma}^{n-1} F_{\gamma} \triangle \tau_{\delta}^{n-1} F_{\delta}\right) \cup \tau_{\delta}\left(\tau_{\gamma}^{n-1} F_{\gamma} \triangle \tau_{\delta}^{n-1} F_{\delta}\right)
$$

Combining with (3) and (21), it follows that for all $n \geq 1$ we have the recurrence relation

$$
\left|\tau_{\gamma}^{n} F_{\gamma} \triangle \tau_{\delta}^{n} F_{\delta}\right| \leq 2 K^{-1}\left(C \varepsilon_{0}+\left|\tau_{\gamma}^{n-1} F_{\gamma} \triangle \tau_{\delta}^{n-1} F_{\delta}\right|\right) .
$$

In particular, (20) and (22) mean that

$$
\sum_{n=0}^{N}\left|\tau_{\gamma}^{n} F_{\gamma} \triangle \tau_{\delta}^{n} F_{\delta}\right| \leq B \varepsilon_{0},
$$

for a constant $B=B(N, C, K)>0$ which is independent of $\gamma$ and $\delta$. Choosing $\varepsilon_{0}=$ $\eta /(2 B)$ establishes (19), as required.

Remark 4.2. For the map $T(x)=2 x(\bmod 1)$, Bousch [Bou1] has given a quantitative bound on the modulus of continuity of the map $\gamma \mapsto e_{F_{\gamma}}$.

COROLlaRY 4.3. The map $\gamma \mapsto \int f^{\prime} e_{F_{\gamma}}$ is continuous.

Proof. For any fixed $g \in L^{\infty}(\mathbb{T})$, the linear functional $L^{1} \rightarrow \mathbb{R}$ defined by $h \mapsto \int g h$ is clearly continuous, with norm $\|g\|_{L^{\infty}}$. Now $\gamma \mapsto e_{F_{\gamma}}$ is continuous, by Proposition 4.1, therefore so is $\gamma \mapsto \int g e_{F_{\gamma}}$, and the result follows by choosing $g=f^{\prime}$. 
We need the following well-known result regarding the invariant measures carried by 1 -flowers.

LEMMA 4.4. Let $T: \mathbb{T} \rightarrow \mathbb{T}$ be an orientation-preserving expanding map.

(a) Every 1-flower carries a unique T-invariant probability measure; any such measure will be called Sturmian.

(b) The support of a Sturmian measure is equal to $\bigcap_{n \geq 0} \overline{\tau^{n}(F)}$, where $\tau$ is either of the two pre-image selectors associated to F.

(c) To each Sturmian measure $S$ there is an associated closed interval $\Gamma_{S}$, such that the 1-flowers carrying $S$ are precisely $\left\{F_{\gamma}\right\}_{\gamma \in \Gamma_{S}}$, where $F_{\gamma}=[a(\gamma), b(\gamma)]$. This closed interval $\Gamma_{S}$ is reduced to a point if and only if $S$ is not a periodic orbit.

Proof. The map $T$ is topologically conjugate to $T_{k}(x)=k x(\bmod 1)($ see $[\mathbf{K H}$, p. 73 , Theorem 2.4.6]), where $k$ is the degree of $T$. The conjugacy sends 1 -flowers of $T$ to 1 -flowers of $T_{k}$ (i.e. closed intervals of length $1 / k$ ), and conjugates the corresponding preimage selectors. The result for $T$ therefore follows from the result for $T_{k}$, and this can be proved by a straightforward adaptation of the approach of either $[\mathbf{B M}]$ or $[\mathbf{B S}]$ for the case $k=2$.

Remark 4.5. (a) Every Sturmian measure is ergodic and the restriction of $T$ to its support is combinatorially equivalent to a rotation (see [BM, BS]). More generally, the dynamics on the maximal closed invariant subset of any flower is combinatorially equivalent to an interval exchange transformation (see [Br1]).

(b) The terminology Sturmian goes back to Morse and Hedlund [MH], who considered certain symbol sequences on a two-letter alphabet. These sequences correspond, under the natural symbolic coding of $T$, to orbits of generic points for our Sturmian measures (see e.g. [Bou1, BM, BS, Jen1]). Some authors (see e.g. [Lot, PF]) prefer the term balanced rather than Sturmian, reserving the term Sturmian for the non-periodic case.

By analogy with Definition 3.1, we introduce the following notions.

Definition 4.6. Let $T: \mathbb{T} \rightarrow \mathbb{T}$ be an expanding map. Recall that the set $\mathcal{M}_{T}$ of $T$-invariant Borel probability measures is compact for the weak* topology. Let

$$
\alpha(f)=\max _{\mu \in \mathcal{M}_{T}} \int f d \mu
$$

denote the maximum ergodic average of the continuous function $f: \mathbb{T} \rightarrow \mathbb{R}$.

The function $f$ is said to be in normal form if $f \leq \alpha(f)$.

Let $F$ be a flower for $T$. We say that $f$ can be continuously maximized on $F$ if there exists a weak coboundary $g: \mathbb{T} \rightarrow \mathbb{R}$ such that the set of global maxima of the function $f+g$ is precisely $F$. We say that $f$ can be Lipschitz maximized on $F$ if there exists a Lipschitz coboundary $g: \mathbb{T} \rightarrow \mathbb{R}$ such that the set of global maxima of the function $f+g$ is precisely $F$ (or, equivalently, there exists a Lipschitz function $\varphi: \mathbb{T} \rightarrow \mathbb{R}$ such that the set of global maxima of $f+\varphi-\varphi \circ T$ is precisely $F$ ).

Remark 4.7. (a) Clearly if $f$ is continuously (respectively Lipschitz) maximized by $F$ then it is continuously (respectively Lipschitz) flattened by $F$. Moreover, if $g$ is 
the corresponding weak coboundary then $f+g$ is in normal form (since $F$ carries a $T$-invariant probability measure, so $\alpha(f)=\max f$ ); therefore the $f$-maximizing measures are precisely those carried by $F$.

(b) If $F$ is a 1-flower then the notion of a function being maximized on $F$ corresponds to the Sturmian condition defined by Bousch [Bou1].

(c) Brémont [Br2] considers Lipschitz functions maximized on flowers, showing that such functions can be Lipschitz approximated by functions with periodic maximizing measures.

The following well-known result guarantees that if $f$ is Lipschitz then we can add a Lipschitz coboundary to it so that the resulting function is in normal form.

Lemma 4.8. Let $T: \mathbb{T} \rightarrow \mathbb{T}$ be an expanding map, and let $f: \mathbb{T} \rightarrow \mathbb{R}$ be Lipschitz. There exists a Lipschitz function $\varphi$ such that $f+\varphi-\varphi \circ T \leq \alpha(f)$.

Proof. This result seems to date back to an unpublished manuscript of Conze and Guivarc'h [CG]. Published proofs can be found in [Bou2, Theorem 1] and [Jen2, Theorem 4.7], while the proofs of [Bou1, Lemma A] and [CLT, Theorem 9], where the stated hypotheses are slightly stronger than ours, are also easily adapted.

THEOREM 4.9. Let $T: \mathbb{T} \rightarrow \mathbb{T}$ be an orientation-preserving Lipschitz expanding map, and let $f: \mathbb{T} \rightarrow \mathbb{R}$ be Lipschitz. If a Sturmian measure $S \in \mathcal{M}_{T}$ is $f$-maximizing, then there exists a 1-flower $F$ carrying $S$ such that $f$ can be Lipschitz flattened on $F$.

Proof. In view of Lemma 4.8, it will suffice to show that if $f \leq \alpha(f)$ then $f$ can be Lipschitz flattened on $F$. So assume that the Lipschitz function $f$ satisfies $f \leq \alpha(f)$, and the Sturmian measure $S$ is $f$-maximizing. Note that such an $f$ is identically equal to $\alpha(f)$ on $\operatorname{supp}(S)$.

By Theorem 3.6, it suffices to show that there exists a 1-flower $F$ containing $\operatorname{supp}(S)$ such that

$$
\int f^{\prime} e_{F}=0
$$

where

$$
e_{F}=\sum_{n \geq 0} \chi\left(\tau_{F}^{n} F\right)
$$

is the escape time function for $F$.

Let $F_{\gamma^{-}}=\left[a\left(\gamma^{-}\right), b\left(\gamma^{-}\right)\right]$denote the 1-flower whose right endpoint is the rightmost point of $\operatorname{supp}(S)$, and $F_{\gamma^{+}}=\left[a\left(\gamma^{+}\right), b\left(\gamma^{+}\right)\right]$the 1-flower whose left endpoint is the leftmost point of $\operatorname{supp}(S)$. Of course, if $S$ is a non-periodic Sturmian measure then $F_{\gamma^{-}}=$ $F_{\gamma^{+}}$is the unique 1-flower containing $\operatorname{supp}(S)$, while if $S$ is periodic then $F_{\gamma^{-}} \neq F_{\gamma^{+}}$.

We claim that

$$
\int f^{\prime} e_{F_{\gamma^{-}}} \geq 0 \text { and } \int f^{\prime} e_{F_{\gamma^{+}}} \leq 0 .
$$

Now $\gamma \mapsto \int f^{\prime} e_{F_{\gamma}}$ is continuous by Corollary 4.3, so once (24) is established, the intermediate value theorem will imply the existence of $\gamma_{0} \in\left[\gamma^{-}, \gamma^{+}\right]$such that (23) holds for $F=F_{\gamma_{0}}=\left[a\left(\gamma_{0}\right), b\left(\gamma_{0}\right)\right]$. 
So to prove the theorem it remains to prove (24). In fact, we shall only prove that $\int f^{\prime} e_{F_{\gamma^{-}}} \geq 0$, as the proof of the other inequality is analogous. To simplify notation we shall write $G=F_{\gamma^{-}}$. We have

$$
\int f^{\prime} e_{G}=\int f^{\prime} \sum_{n \geq 0} \chi\left(\tau_{G}^{n} G\right)=\sum_{n \geq 0} \int_{\tau_{G}^{n} G} f^{\prime} .
$$

Now $G$ is an interval, and $\tau_{G}$ has a single jump discontinuity, so each $\tau_{G}^{n} G$ is a union of $s_{n} \leq n+1$ intervals $I_{j}^{(n)}$, which we write as

$$
\tau_{G}^{n} G=\bigcup_{j=1}^{s_{n}} I_{j}^{(n)} .
$$

Let $c_{j}^{(n)}$ and $d_{j}^{(n)}$ denote, respectively, the left and right endpoints of $I_{j}^{(n)}$. The right endpoint of $G$ is a point in $\operatorname{supp}(S)$; therefore every right endpoint $d_{j}^{(n)}$ is also a point in $\operatorname{supp}(S)$. It follows that

$$
f\left(d_{j}^{(n)}\right)=\alpha(f)=\max f \geq f\left(c_{j}^{(n)}\right) .
$$

Now $f$ is Lipschitz, hence absolutely continuous, so applying the fundamental theorem of calculus, and then (26), gives

$$
\int_{c_{j}^{(n)}}^{d_{j}^{(n)}} f^{\prime}=f\left(d_{j}^{(n)}\right)-f\left(c_{j}^{(n)}\right) \geq 0 .
$$

Therefore for all $n \geq 0$,

$$
\int_{\tau_{G}^{n} G} f^{\prime}=\sum_{i=1}^{s_{n}} \int_{c_{j}^{(n)}}^{d_{j}^{(n)}} f^{\prime} \geq 0,
$$

so from (25) we deduce that

$$
\int f^{\prime} e_{G}=\sum_{n \geq 0} \int_{\tau_{G}^{n} G} f^{\prime} \geq 0,
$$

as required.

Remark 4.10. As mentioned in $\S 1$, for certain functions $f$ the maximizing measure is known to be Sturmian, but a priori it is not known which of the Sturmian measures is maximizing. A consequence of Theorem 4.9 is that, in order to show that a particular Sturmian measure $S$ is $f$-maximizing, it suffices to locate a 1-flower $F$ which carries $S$ and on which $f$ can be Lipschitz flattened. If $\gamma \mapsto F_{\gamma}$ is a parametrization of the 1-flowers for $T$ then, by Theorem 3.6, we must find $\gamma$ such that

$$
\int e_{F_{\gamma}} f^{\prime}=0
$$

Equation (27) can be solved numerically $\dagger$ by approximating the escape time functions $e_{F_{\gamma}}=\sum_{n=0}^{\infty} \chi\left(\tau_{F_{\gamma}}^{n}\left(F_{\gamma}\right)\right)$ by finite truncations $e_{F_{\gamma}, N}:=\sum_{n=0}^{N} \chi\left(\tau_{F_{\gamma}}^{n}\left(F_{\gamma}\right)\right)$. The distance $\dagger$ An approximate solution is typically sufficient, in view of the fact that periodic Sturmian measures are carried by a parameter interval of 1 -flowers. 
$\left\|e_{F_{\gamma}}-e_{F_{\gamma}, N}\right\|_{L^{1}}$ decreases exponentially with $N$, so solutions to the equation $\int e_{F_{\gamma}, N} f^{\prime}=0$, which can be computed using a root-finding algorithm such as Newton's method, converge to solutions of (27) at an exponential rate.

Question 4.11. Does some analogue of Theorem 4.9 hold for more general flowers? For example, suppose $\mu$ is the unique invariant measure carried by some flower $F$, and is the unique maximizing measure for a Lipschitz function $f$. Is it then the case that $f$ can be Lipschitz flattened on some flower (not necessarily $F$ ) which carries $\mu$ (and which necessarily carries no other invariant measure)? The proof of any such result would seem to require a higher-dimensional analogue of the intermediate value theorem.

This question also raises the issue of whether or not every invariant measure carried by some flower is in fact the unique invariant measure carried by some (other) flower.

Example 4.12. If a Lipschitz function $f$ has a Sturmian maximizing measure $S$, Theorem 4.9 guarantees that $f$ can be Lipschitz flattened on some 1-flower which carries $S$. One might expect that in fact such a 1-flower Lipschitz maximizes $f$, in the sense of Definition 4.6 (e.g. this is exactly what Bousch [Bou1] proves in the case where $T(x)=2 x$ ( $\bmod 1)$ and $f$ is a trigonometric polynomial of degree one). However, in general this is not the case: for example if $f$ has a Sturmian maximizing measure, but this is not the unique $f$-maximizing measure, then clearly no 1 -flower can maximize $f$. In fact even when the Sturmian measure is the unique maximizing measure, it is not the case that there exists a 1-flower which maximizes $f$, as the following example shows.

Consider the expanding map $T(x)=2 x(\bmod 1)$, whose 1 -flowers are precisely the set of all closed semi-circles (i.e. intervals of length $1 / 2$ ). There are infinitely many $\gamma \in(0,1 / 6)$ such that the 1 -flower $F_{\gamma}=[\gamma, \gamma+1 / 2]$ contains a non-periodic Sturmian measure $S$ (see e.g. [BS]). Fix one such $\gamma$, and let $\tau$ denote the pre-image selector for $F_{\gamma}$ defined by

$$
\tau(x)= \begin{cases}(x+1) / 2 & \text { if } x \in[0,2 \gamma), \\ x / 2 & \text { if } x \in[2 \gamma, 1) .\end{cases}
$$

Since $\operatorname{supp}(S)$ is the intersection of the decreasing sequence $\overline{\tau^{n}\left(F_{\gamma}\right)}$, the Sturmian measure $S$ is in particular carried by

$$
\overline{\tau\left(F_{\gamma}\right)}=[\gamma, \gamma / 2+1 / 4] \cup[\gamma / 2+1 / 2, \gamma+1 / 2] .
$$

Let $f: \mathbb{T} \rightarrow \mathbb{R}$ be the continuous piecewise linear function (see Figure 3 ) whose maximum value is 0 and whose derivative is given by

$$
f^{\prime} \equiv \begin{cases}0 & \text { on }(\gamma, \gamma / 2+1 / 4), \\ -2 / \gamma & \text { on }(\gamma / 2+1 / 4, \gamma+1 / 4), \\ 2 /(1 / 2-\gamma) & \text { on }(\gamma+1 / 4, \gamma / 2+1 / 2), \\ 0 & \text { on }(\gamma / 2+1 / 2, \gamma+1 / 2), \\ -1 & \text { on }(\gamma+1 / 2, \gamma+3 / 4), \\ 1 & \text { on }(\gamma+3 / 4, \gamma) .\end{cases}
$$




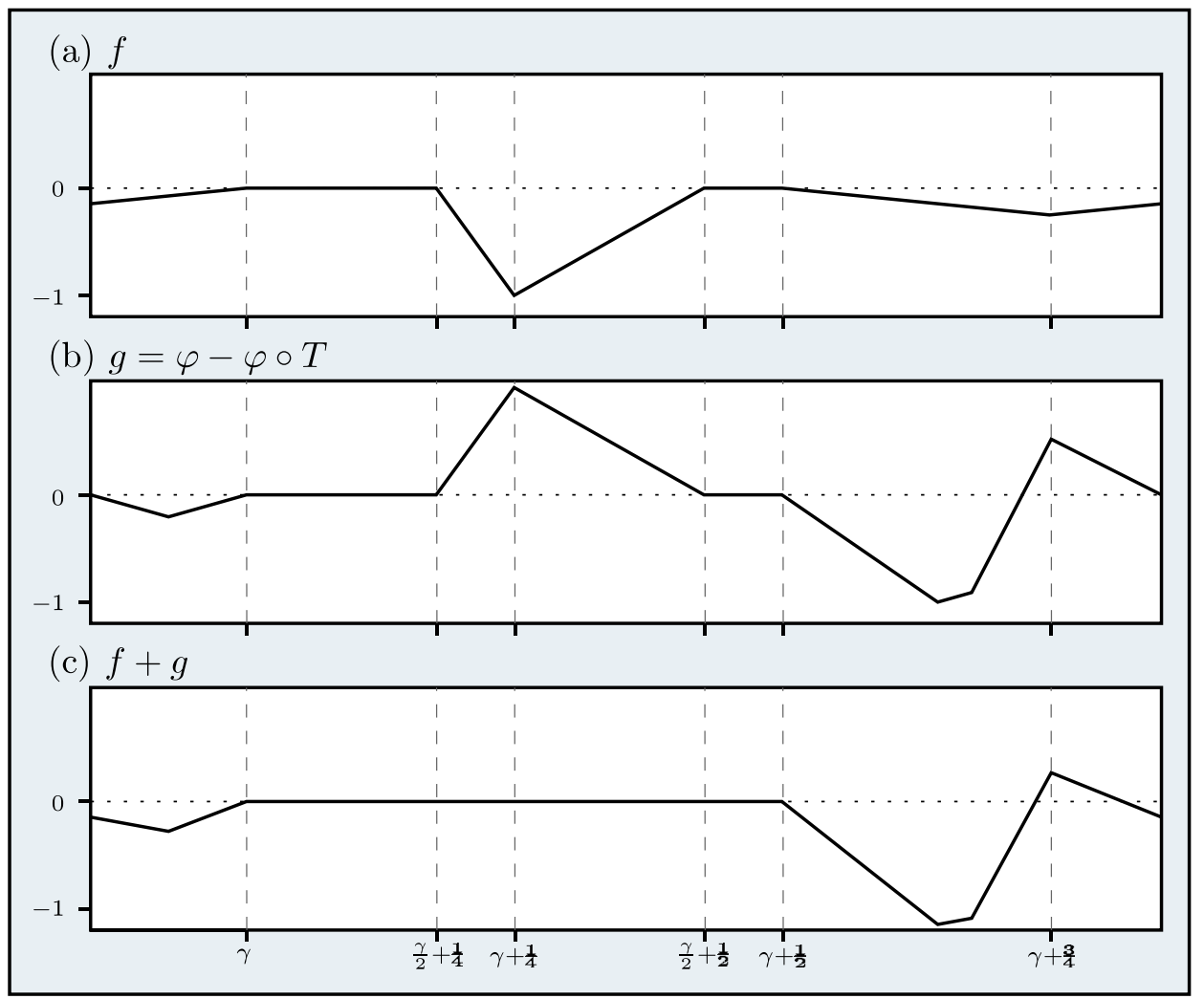

FIGURE 3. Flattening of the function $f$ from Example 4.12: $f$ itself is shown in (a), the unique Lipschitz coboundary $g=\varphi-\varphi \circ T$ flattening $f$ on the 1 -flower $F=[\lambda, \lambda+1 / 2]$ is shown in (b), and the flat function $f+g$ is shown in (c). Although flat on $F$, the function $f+g$ is not in normal form as its maximum (at $\gamma+3 / 4$ ) is larger than 0 . The function $f$ is in normal form; the unique Sturmian measure carried by $F$ is also carried by the set of maxima of $f$, so is the unique $f$-maximizing measure. Thus $f$ may be flattened on $F$ (i.e. satisfies the pre-Sturmian condition), but the corresponding flat function is not in normal form, so $f$ does not satisfy the Sturmian condition, even though its maximizing measure is Sturmian.

Note that $f$ is in normal form: its set of global maxima

$$
f^{-1}(0)=[\gamma, \gamma / 2+1 / 4] \cup[\gamma / 2+1 / 2, \gamma+1 / 2]
$$

carries the Sturmian measure $S$, and this is the unique maximizing measure. Now $F_{\gamma}$ is the only 1-flower which contains $S$, so if $f$ is Lipschitz maximized by a 1-flower then it must be Lipschitz maximized by $F_{\gamma}$. There is a unique Lipschitz coboundary $g$ such that $\left.(f+g)\right|_{F_{\gamma}}$ is a constant (cf. Remark 3.7), so to show that $f$ is not Lipschitz maximized by $F_{\gamma}$ it suffices to show that $F_{\gamma}$ is not the set of maxima of the function $f+g$.

Let $\varphi: \mathbb{T} \rightarrow \mathbb{R}$ be a continuous piecewise linear function (uniquely defined up to an additive constant) whose derivative is given by 


$$
\varphi^{\prime} \equiv \begin{cases}0 & \text { on }(\gamma, \gamma+1 / 2) \\ -1 / \gamma & \text { on }(\gamma+1 / 2,2 \gamma+1 / 2) \\ 1 /(1 / 2-\gamma) & \text { on }(2 \gamma+1 / 2, \gamma),\end{cases}
$$

and define the Lipschitz coboundary $g$ by

$$
g=\varphi-\varphi \circ T
$$

It is readily verified that

$$
\left.(f+g)\right|_{F_{\gamma}} \equiv 0 .
$$

However,

$$
\begin{aligned}
(f & +g)(\gamma+3 / 4)=(f+g)(\gamma+3 / 4)-(f+g)(\gamma+1 / 2) \\
& =\int_{\gamma+1 / 2}^{\gamma+3 / 4}(f+g)^{\prime} \\
& =\int_{\gamma+1 / 2}^{2 \gamma+1 / 2}(f+g)^{\prime}+\int_{2 \gamma+1 / 2}^{\gamma / 2+3 / 4}(f+g)^{\prime}+\int_{\gamma / 2+3 / 4}^{\gamma+3 / 4}(f+g)^{\prime} \\
& =-\gamma\left(1+\frac{1}{\gamma}\right)+\left(\frac{1}{4}-\frac{3 \gamma}{2}\right)\left(\frac{1}{1 / 2-\gamma}-1\right)+\frac{\gamma}{2}\left(\frac{1}{1 / 2-\gamma}+\frac{2}{\gamma}-1\right) \\
& =\frac{1}{4}-\frac{\gamma}{1-2 \gamma},
\end{aligned}
$$

which is strictly positive because $\gamma \in(0,1 / 6)$. So 0 is not the maximum value of $f+g$, and therefore $F_{\gamma}$ is not the set of maxima of $f+g$. Therefore $F_{\gamma}$ does not Lipschitz maximize $f$, as required.

Acknowledgements. Both authors were partially supported by EPSRC grant GR/S50991/01. The second author was partially supported by an EPSRC Advanced Research Fellowship.

\section{REFERENCES}

[ADJR] V. Anagnostopoulou, K. Díaz-Ordaz, O. Jenkinson and C. Richard. Escape from a semi-circle and Sturmian maximizing measures. Preprint, 2007.

[Bou1] T. Bousch. Le poisson n'a pas d'arêtes. Ann. Inst. H. Poincaré Probab. Statist. 36 (2000), 489-508.

[Bou2] T. Bousch. La condition de Walters. Ann. Sci. École Norm. Sup. (4) 34 (2001), 287-311.

[BJ] T. Bousch and O. Jenkinson. Cohomology classes of dynamically non-negative $C^{k}$ functions. Invent. Math. 148 (2002), 207-217.

[BM] T. Bousch and J. Mairesse. Asymptotic height optimization for topical IFS, tetris heaps, and the finiteness conjecture. J. Amer. Math. Soc. 15 (2002), 77-111.

[Br1] J. Brémont. Dynamics of injective quasi-contractions. Ergod. Th. \& Dynam. Sys. 26 (2006), 19-44.

[Br2] J. Brémont. Finite flowers and maximizing measures for generic Lipschitz functions on the circle. Nonlinearity 9 (2006), 813-828.

[BS] S. Bullett and P. Sentenac. Ordered orbits of the shift, square roots, and the devil's staircase. Math. Proc. Cambridge Philos. Soc. 115 (1994), 451-481.

[CG] J.-P. Conze and Y. Guivarc'h. Croissance des sommes ergodiques. Manuscript, circa 1993. 
[CLT] G. Contreras, A. O. Lopes and Ph. Thieullen. Lyapunov minimizing measures for expanding maps of the circle. Ergod. Th. \& Dynam. Sys. 21 (2001), 1379-1409.

[Jen1] O. Jenkinson. Frequency locking on the boundary of the barycentre set. Experiment. Math. 9 (2000), 309-317.

[Jen2] O. Jenkinson. Ergodic optimization. Discrete Contin. Dyn. Syst. Ser. B 15 (2006), 197-224.

[Jen3] O. Jenkinson. Optimization and majorization of invariant measures. Electron. Res. Announc. Amer. Math. Soc. 13 (2007), 1-12.

[Jen4] O. Jenkinson. A partial order on $\times 2$-invariant measures. Preprint, 2006.

[KH] A. Katok and B. Hasselblatt. Introduction to the Modern Theory of Dynamical Systems. Cambridge University Press, Cambridge, 1995.

[Liv] A. Livšic. Homology properties of $Y$-systems. Mat. Zametki 10 (1971), 758-763.

[Lot] M. Lothaire. Algebraic Combinatorics on Words (Encyclopaedia of Mathematics and its Applications, 90). Cambridge University Press, Cambridge, 2002.

[MH] M. Morse and G. A. Hedlund. Symbolic dynamics II. Sturmian trajectories. Amer. J. Math. 62 (1940), 1-42.

[PF] N. Pytheas Fogg. Substitutions in Dynamics, Arithmetics and Combinatorics (Lecture Notes in Mathematics, 1794). Springer, Berlin, 2002.

[Zie] W. Ziemer. Weakly Differentiable Functions. Springer, Berlin, 1989. 